\title{
Generation of Open Metatarsal Fracture in Rats: A Model for Secondary Fracture Healing
}

\author{
Abubakar Adamu Abdul a,d, Ahmed Khalaf Ali a,e , Sahar Mohammad Ibrahim ${ }^{\text {a,e }}$, \\ Kareem Obayes Handool a, Mohammad Shoaib Khan a,f Mustapha Mohamed Noordin ${ }^{\text {b }}$, \\ Tengku-Azmi Tengku Ibrahim ${ }^{c}$, Ubedullah Kaka ${ }^{\text {a }}$, Loqman Mohamad Yusof ${ }^{\text {a* }}$ \\ ${ }^{a}$ Department of Companion Animal Medicine and Surgery, Faculty of Veterinary Medicine, Universiti Putra Malaysia, 43400 \\ Universiti Putra Malaysia, Serdang, Malaysia. \\ ${ }^{b}$ Department of Veterinary Pathology and Microbiology, Faculty of Veterinary Medicine, Universiti Putra Malaysia, 43400 Universiti \\ Putra Malaysia, Serdang, Malaysia. \\ ${ }^{c}$ Department of Pre-Clinical Veterinary Studies, Faculty of Veterinary Medicine, Universiti Putra Malaysia, 43400 Universiti Putra \\ Malaysia, Serdang, Malaysia. \\ ${ }^{d}$ Department of Veterinary Surgery and Radiology, Usmanu Danfodiyo University, Sokoto, Nigeria. \\ ${ }^{e}$ Department of Surgery and Theriogenology, College of Veterinary Medicine, University of Mosul, Iraq. \\ ${ }^{f}$ Faculty of Veterinary and Animal Science, Gomal University, DI. Khan, Pakistan. \\ Received 26 July 2020; Accepted 22 October 2020
}

\begin{abstract}
A fracture model in rats for the study of secondary bone healing was described. Standard open midshaft transverse metatarsal fracture was produced with bone cutting forceps in 28 rats. The commonly open and close fracture models utilized for bone and mineral researches are associated with varying degree of complications ranging from a high degree of fracture comminution to severe associated soft tissue injury which interferes with the healing process. We hypothesized that fracture model in rat third metatarsal bone could be associated with low -post-surgical complications and could be a reproducible model. To test this, open mid-shaft transverse fractures were created on the metatarsals of 28 rats. The objectives of the study were to evaluate the fracture complications, to determine the nature of fracture produced, evaluate the fracture consolidation during healing periods, and to assess the histological and radiographic healing of the fracture. The fracture produced in the mid metatarsal shaft of all rats was $100 \%$ transverse, $73 \%$ located at the midshaft. Minimal fracture angulations were recorded $\left(0.48 \pm 0.09^{\circ} ; 0.78 \pm 0.17^{\circ}\right)$ for anterior-posterior and lateral views respectively. Minimal soft tissue injury was recorded immediately post-surgery, but no infection and the delayed union was observed. Varying degrees of weight-bearing lameness was also recorded but seized at day six onward postoperative. Callus index observed was peaked at week 2 and $3(2.02 \pm 0.1,1.99 \pm 0.13)$ respectively but declined to $1.10 \pm$ 0.04 at week 7 during the consolidation period. The fracture line disappeared completely at week 7 . The histological and radiographic healing scores were $(3.5 \pm 0.13$ and $3.75 \pm 0.25)$ respectively (out of the maximum healing score of 4$)$ at week 7 post-operative. There was a positive correlation between the histological and radiographic healing scores. The metatarsal fracture model is considered to be a suitable model for in vivo study of secondary fracture healing.
\end{abstract}

Keywords: Fracture Model; Rat; Fracture Healing; Metatarsal.

\section{Introduction}

Fracture healing is a complex, well organized sequential physiological process, involving cellular and molecular events that still need to be explored [1]. To investigate bone healing in an organized manner, numerous pre-clinical

* Corresponding author: loqman@upm.edu.my

doi http://dx.doi.org/10.28991/SciMedJ-2020-0204-2

$>$ This is an open access article under the CC-BY license (https://creativecommons.org/licenses/by/4.0/).

(C) Authors retain all copyrights. 
laboratory models have been developed and utilized extensively [2]. The use of laboratory models becomes essential to enhance the understanding of the mechanism involved in fracture healing, as such various laboratory animal models (avian species, goat, sheep, dog, pig and monkey) were tested in numerous fracture healing investigations [3-6]. Currently, the focus is on the use of small laboratory animals such as mouse, rat and rabbits because they are cheaper, easy to handle, and can be genetically modified [7-10].

The previous model of bone fracture repair in rodents were produced by the application of blunt trauma externally on the desired long bone (usually femur or tibia) with the aim of generating a standardized mid-shaft, closed, simple transverse fracture. This model was first designed by Jackson et al. [11] and fully described by Bonnaren and Einhorn [12] using three-point bending device known as the guillotine. The model was widely accepted for the generation of rodent fracture model by numerous biomedical investigators [13, 14]. Over the years, there was a modification of this model due to some associated limitations $[15,16]$. One of the modifications was developed by Otto et al. [17] where closed femoral fracture was generated in a rat model using modified three-point bending device utilizing small ruminants ear tag applicator with the hope to minimized soft tissue damage associated with the blunt guillotine method. However, this model was not widely accepted in comparison with the previous Bonnaren and Einhorn model.

To date, the major challenge in the generation of closed fracture model using the blinded three-point bending device are a series of complications associated with soft tissue damage, high degrees of fracture communition, fracture angulations, mal-union, displacement, infection and death not related to anaesthesia [15, 17]. In this model, the generation of the fracture was carried out by blindly throwing a blunt object of defined mass, usually at a given distance and force, which no doubt creates severe injury to the soft tissue surrounding the bone, thereby interfering with the fracture healing process as reported by Auregan et al. [15] and Handool et al. [10]. These limitations made the reproducibility of this model inconsistent to many investigators.

Experimental fracture models equip investigators to test different research interventions on the normal processes of bone healing $[18,19]$. Most of the studies utilize rodents for fracture models, because it has been reported that fracture healing in rodents is extremely rapid and effective when compared to typical clinical scenarios [20]. Therefore, researchers must be cautious in translating outcomes related to time of fracture union or stiffness of the union in relation to clinical benefit [18]. A considerable number of fracture models were described in the literatures, with the variations of the models are typically defined by the animal used, the site of the fracture and the pattern whether it is open or closed. Other consideration is whether the animal used was genetically manipulation or not [21, 22].

Traditionally, the use of large animals for fracture healing model was preferred because bone remodeling in large animals have been reported to mimic the scale and structure of the human bone [22]. However, with the advent of molecular and genetic techniques, the use of laboratory animal models has become popular [23]. The murine fracture healing models is readily amenable to genetic alteration and could be studied with existing antibodies and probes. Another advantage of the laboratory animal model is the ability to maintain the animals at relatively low costs when compared to large-animal models [19].

The soft tissue injuries associated with the severe open fractures is one of the great challenges for both open and closed fracture models [24]. Although the severity of the closed fracture model is greater than that of the open fracture model. The soft tissue injury may lead to numerous complications, such as osteomyelitis, tissue necrosis, ischemia, and limb amputation in some cases [23, 35]. It is widely accepted that the outcomes of fracture healing depends not only on the fracture itself but also the combined soft tissue injury [22]. Therefore, it is important to develop an animal model for open fracture research with minimal soft tissue injury in order to overcome the challenging factors that may interfere with the fractures healing.

This study was aimed at developing an improved quality and reproducible experimental fracture model on the metatarsal of rat with minimal complications. To achieve this, standard mid-shaft transverse fracture was generated at the third metatarsal bone using Liston bone cutting forceps (Solingen, Germany). After generation of the fracture, the following parameters were used to assess the fracture model viz; evaluation of the fracture complications, determination of the pattern of fracture produced, evaluation of the fracture consolidation during healing and assessment of the histological and radiographic healing of the fracture produced. The choice of third metatarsal bone was considered for two major reasons; we have in mind that the two left and right lateral metatarsal bones $\left(2^{\text {nd }}\right.$ and $\left.4^{\text {th }}\right)$ could help stabilized the fracture without the required fixation with an intramedullary pin or any other bone implants and to avoid anatomical areas where there is excessive soft tissue mass, to minimized soft tissue damage interfering with the fracture healing. To the best of our knowledge, this is the first fracture model of secondary bone healing using the third metatarsal, which is considered as one of the long bones. The outcome of this investigation may be useful to researchers to efficiently study the bone healing, genetic modification of fracture healing, enhancement of fracture healing, novel treatments on bone healing and other relevant clinical applications involving rodent fracture model. 


\section{Materials and Methods}

\subsection{Research Animals and Experimental Design}

The study was undertaken according to the protocol approved by the Institutional Animal Care and Used Committee of Universiti Putra Malaysia (IACUC, UPM) (reference no. R028/2015). Twenty-eight (28) female Sprague-Dawley rats were acquired from animal resource facility of the Faculty of Veterinary Medicine, Universiti Putra Malaysia (ARF, UPM). The animals were six weeks old with a weight range between 190-220g, with no more than 40 grams variation in group weight. They were housed and fed according to the principles and guideline of the IACUC, UPM. The rats were conditioned for one week before the start of the experiment. The summary of the experimental design is provided in Figure 1 below.

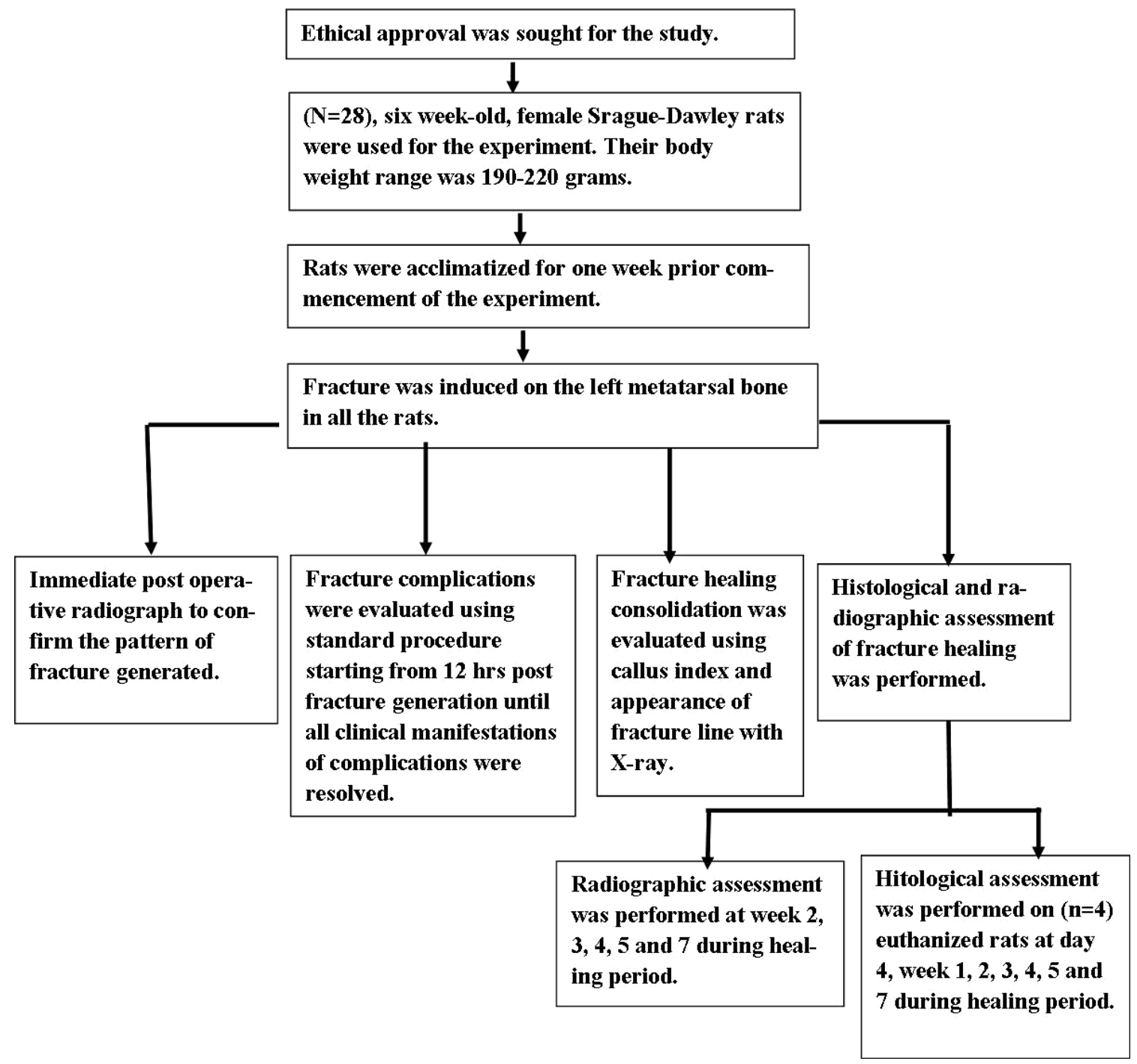

Figure 1. Flowchart of the experimental design, from the ethical approval to completion of the study

Each rat was anaesthetised using intramuscular injections of a mixture of ketamine (Ketamine injection, 100 $\mathrm{mgmL}^{-1}$, Troy laboratories PTY, Australia) and xylazine (Ilium Xylazil $100 \mathrm{mgmL}^{-1}$, Troy laboratories PTY, Australia) $70 \mathrm{mgkg}^{-1}$ and $7 \mathrm{mgkg}^{-1}$ respectively. Tramadol $\mathrm{HCl} 50 \mathrm{mg}$ (Biolab Co., LTD, Thailand), was administered subcutaneously at ten $\mathrm{mgkg}_{-}{ }^{1}$ for pre-operative analgesia. The left limb was used for the creation of the fracture in all the rats. The whole metatarsal area was clipped and scrubbed with $4 \%$ chlorhexidine gluconate (Global trade laboratory Co. Ltd, Malaysia), 70\% isopropyl alcohol (Global trade laboratory Co. Ltd, Malaysia), and finally wipe with gauze containing povidone-iodine (Advanced Healthcare Solution, Malaysia) as described by Yakubu et al. [26]. The rats were placed on dorsal position on the surgical table spread with a warm sterile towel.

\subsection{Surgical Procedure}

Standard $1.5 \mathrm{~cm}$ incision was made directly on the third metatarsal of the left limb. The bundles of the ligaments covering the bones were exposed and carefully retracted laterally to expose the bone. The third metatarsal bone was 
identified and cut transversely with Liston bone cutting forceps (Solingen, Germany) at the mid diaphyseal region of the bone. The bone was anatomically reduced before skin closure. The skin was closed in a single layer using a simple interrupted suture pattern with non-absorbable suture Ethilon ${ }^{\mathrm{TM}}$ size 4/0 (Johnson Company, Scotland) (Figure 2).

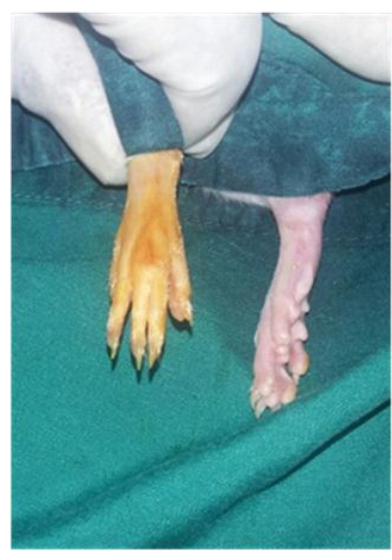

(A)

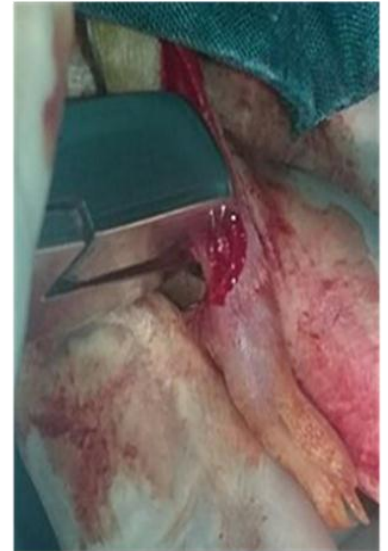

(D)

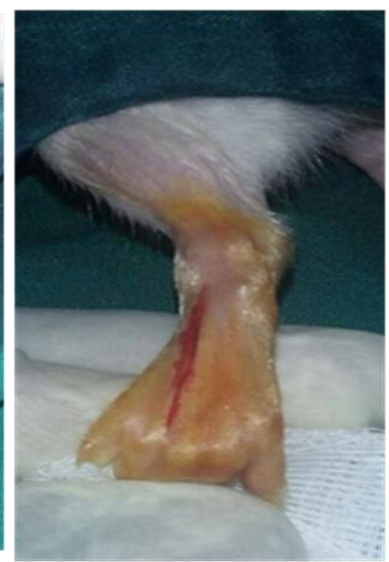

(B)

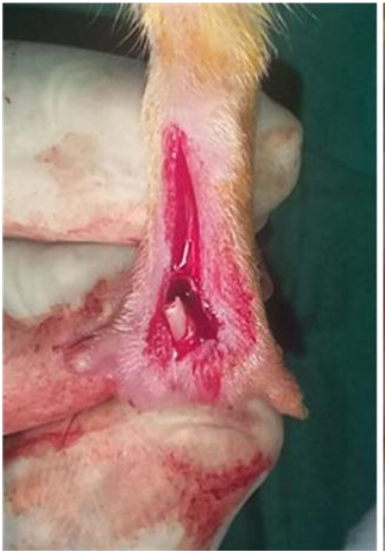

(E)

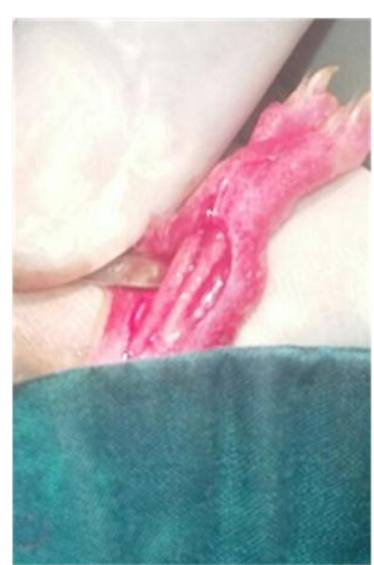

(C)

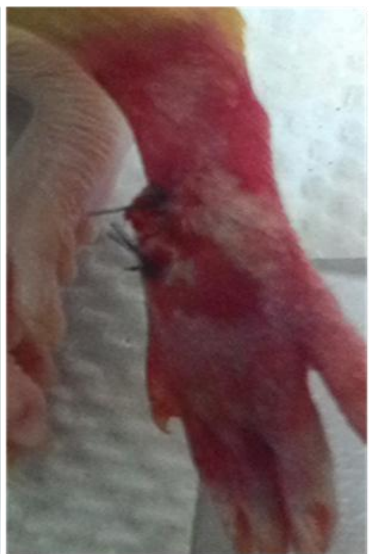

(F)

Figure 2. Photographs of sequential surgical steps of creating 3rd metatarsal transverse mid shaft fracture. (A) final skin preparation with povidone iodine and drapping of the surgical site, (B) skin incision directly on the 3rd metatarsal bone, (C) exposure of the metatarsal bones, (D) fracture creation with Liston bone cutting forceps, $(E)$ transverse mid shaft fracture created, and (F) single layered skin closure with ethilon non absorbable suture in simple interrupted closure pattern after anatomical reduction of the fractured bone

Tramadol $\mathrm{HCl} 50 \mathrm{mgmL}^{-1}$ at ten $\mathrm{mgkg}^{-1}$ was administered subcutaneously for pre and -post-operative analgesia; it was repeated after every $24 \mathrm{hrs}$ for three days. Two standard orthogonal radiographic views lateral (L) and anteriorposterior (AP) of the fractured limb were taken immediately postoperative in order to ascertain the nature and pattern of the fracture produced. The radiographs were taken using portable digital x-ray unit PXP-20HF (Poskom Co., Ltd, Korea) attached with Carestream DRX-mobile retrofit kits (Carestream Health, Inc., USA). The counter lateral right limbs were used as the control in all rats.

The radiograph was repeated at 2, 3, 4, 5 and 7 weeks post-surgery to monitor the progress of the healing. Four $(\mathrm{n}=4)$ rats were euthanized at day 4, then week 1, 2, 3, 4, 5, and 7 post-surgery. Euthanasia was achieved using intraperitoneal injection of $20 \%$ Pentobarbital (Dolethal ${ }^{\circledR}$; Vetoquinol, France) at $90 \mathrm{mgkg}^{-1}$.

\subsection{Evaluation of Fracture Complications}

Complications associated with this fracture model were assessed based on the following parameters; the survival rate of the rats after full recovery from anaesthesia, feed and water intakes, the severity of weight-bearing lameness, degree of soft tissue damage by visual examinations $12 \mathrm{hrs}$. post-surgery until clinical signs were resolved and delayed union using radiographic evidence at the termination of the experiment.

The percentage survival rate was determined by calculating the number of rats that survived the surgical manipulation without mortality associated with anaesthesia complication throughout the period of investigation. Daily feed and water intake per cage were quantified during the one-week conditioning period. This was achieved by giving known quantity of feed and water to the four rats in each cage. After 24 hrs, the leftover quantity was subtracted from the initial amount given to determine the approximate quantity consumed as described by Vento et al. [27]. This 
procedure was carried out for one week prior to fracture induction, and the average feed consumed per cage was computed. The percentage feed and water intake were then determined when rats were returned to their cages after recovery from general anaesthesia on a daily basis until the feed and water intakes were restored to normal.

The severity of weight-bearing lameness was quantified using modified three-point scoring criteria described by Fitzpatrick et al. [28], i.e. rat without evidence of lameness (score 0), partial lameness (score 1), and complete lameness (score 2). The weight-bearing scoring commences immediately within the first 12 hrs. after recovery from anaesthesia and subsequently repeated after every $24 \mathrm{hrs}$. until when the weight-bearing lameness is restored to normal. In this scoring criterion, a rat can only have a single score at a point; therefore maximum score point was 2 while the minimum was 0 .

The degree of soft tissue damage on the other hand was assessed based on visual and physical examinations of the skin surrounding the fractured limb of the rats post-surgery. The evaluation was carried out within the first 12 hrs. post-surgery, and subsequently on a daily basis until clinical signs indicating soft tissue damage disappeared. The soft tissue damage was scored using modified four-point scoring criteria described by Sylvestre et al. [29]. Clinical parameters considered for the scoring were; no visible lesions (score 0), erythema (score 1), visible swelling (score 2), and tissue necrosis (score 3 ). In this scoring criterion, one rat may have all the clinical parameters at a given point; therefore maximum scoring point could reach up to 6 points, while the minimum score could be 0 .

Delayed union as a complication was also assessed using radiographic evidence at the terminal point of the experiment (week seven post-surgery). Week seven was considered a terminal point of the experiment based on the result of the pilot study conducted. The fracture was considered to have delayed union when radiographic evidence shows persistent fracture line at 7-week post-surgery as described by Gomez-Barrena et al. [30].

\subsection{Assessment of the Pattern of Fracture Produced}

The pattern and consistency of fracture created was evaluated to determine if the fracture is transverse, spiral or oblique with or without communition. The anatomical location of the fracture was also assessed and determined using radiographic evidence within $24 \mathrm{hrs}$. post-surgery. Fracture angulations were also quantified as described by Lamraski et al. [31] and Auregan et al. [15] using radiographic evidence with Image J software version 1.50d (NIH, Maryland; USA).

\subsection{Evaluation of Fracture Consolidation}

Fracture consolidation was assessed and evaluated based on two parameters; callus index and fracture line disappearance in the course of the healing period. The callus index was measured by taking the ratio of the callus diameter against the diameter of the counter lateral normal limb (callus diameter/normal bone diameter) as described by Porter et al. [32]. The callus diameter was measured under the dissecting stereo-microscope (Huvitz; HSZ-645TR, Korea) fitted with 3.1 mega pixel digital camera (VIS imaging; UC3010; Malaysia) and VIS plus ver.3.50 image analysis and measurement software; Canada. The diameters of the callus and normal bones were measured in millimeter $(\mathrm{mm})$ at x 6.5 objective magnification according to the standard procedure described by Abubakar et al. [33]. The radiographic evaluation fracture line disappearance was determined, according to Auregan et al. [15] at different radiographic evaluation periods of the study.

\subsection{Histological and Radiographic Assessment of Healing}

Both fractured and the corresponding counter lateral metatarsal bones were dissected and harvested after the rats were euthanized at different healing intervals, as indicated earlier. The bones were decalcified with $10 \%$ formic acid (Sigma-Aldrich, USA) for four days, every $24 \mathrm{hrs}$., the formic acid solution was discarded and replaced with a new stock solution and the bones were fixed in $10 \%$ buffered formalin (Sigma-Aldrich, USA). They were dehydrated through a series of ethanol solution using (TP 1020 semi-closed benchtop tissue processor; Leica, Singapore), then embedded in paraffin wax using standard procedure described by Duttmeyer [34]. Four (4 $\mu$ m) serial sections were obtained using Reichert-Jung 2045 multicut rotary microtome, USA. The sections were then mounted on poly-Llysine coated microscope slides (Menzel-Glaser, Germany). After de-parafinization with xylene and rehydration with decreasing concentration of ethanol solution $(100 \%$ and $70 \%)$ the sections were stained with hematoxylin and eosin $(\mathrm{H}$ and E), air-dried and mounted with a cover slip.

The histological and radiographic assessment score was carried out by two independent assessors that were blinded to the experimental design. The histological slides were assessed and healing was scored at week seven when the experiment was terminated, healing was scored using five-point modified scoring criteria described by Shoji et al. [35]. The histological features used for the scoring were; pseudoarthrosis formation (score 0), incomplete cartilaginous union with fibrous remnant within callus (score 1), complete cartilage union (score 2), bony union less than complete due to presence of a small amount of cartilage in the callus (score 3), and complete bony union (score 4). 
Radiographic features of healing were assessed and scored using modified five-point grading criteria described by Estai et al. [36]. The radiographic assessment score was carried out by two independent assessors that were blinded to the experimental design. The radiographic features used for the scoring were; no evidence of healing (score 0), evidence of callus formation but fracture gap not bridged (score 1), callus formation evidence with a possible bridge of fracture gap (score 2), callus formation evidence with the good bridging of fracture gap (score 3), evidence of fracture union (score 4).

\subsection{Data Analysis}

Data were presented in the form of tables and graphs. Comparison between two sets of data was carried out using Student's $t$-test or a suitable non-parametric test if the data set were not normally distributed. Time-course experiments were analyzed with a one-way ANOVA for which appropriate post hoc-tests for comparisons was conducted. The analysis was carried out using IBM SPSS for Windows, version 22.0, while graphs were plotted using GraphPad Prism 7. Non-scoring data were presented as mean and standard error of the mean (SEM). $P$ values $<0.05$ were considered significant.

\section{Results}

\subsection{Fracture complications}

All the 28 rats $(100 \%)$ operated survived to their allocated euthanasia period; there was no case of infection of the surgical site recorded. Feed and water intake were reduced by $20 \%$ within the first 72 hrs., and by $10 \%$ within 96 hrs. post-surgery. Normal feed and water intake were restored at six days post-surgery. Radiographic and histological results at fifth and seventh weeks post-surgery indicate no evidence of delayed union at the fracture site (Figure 3).

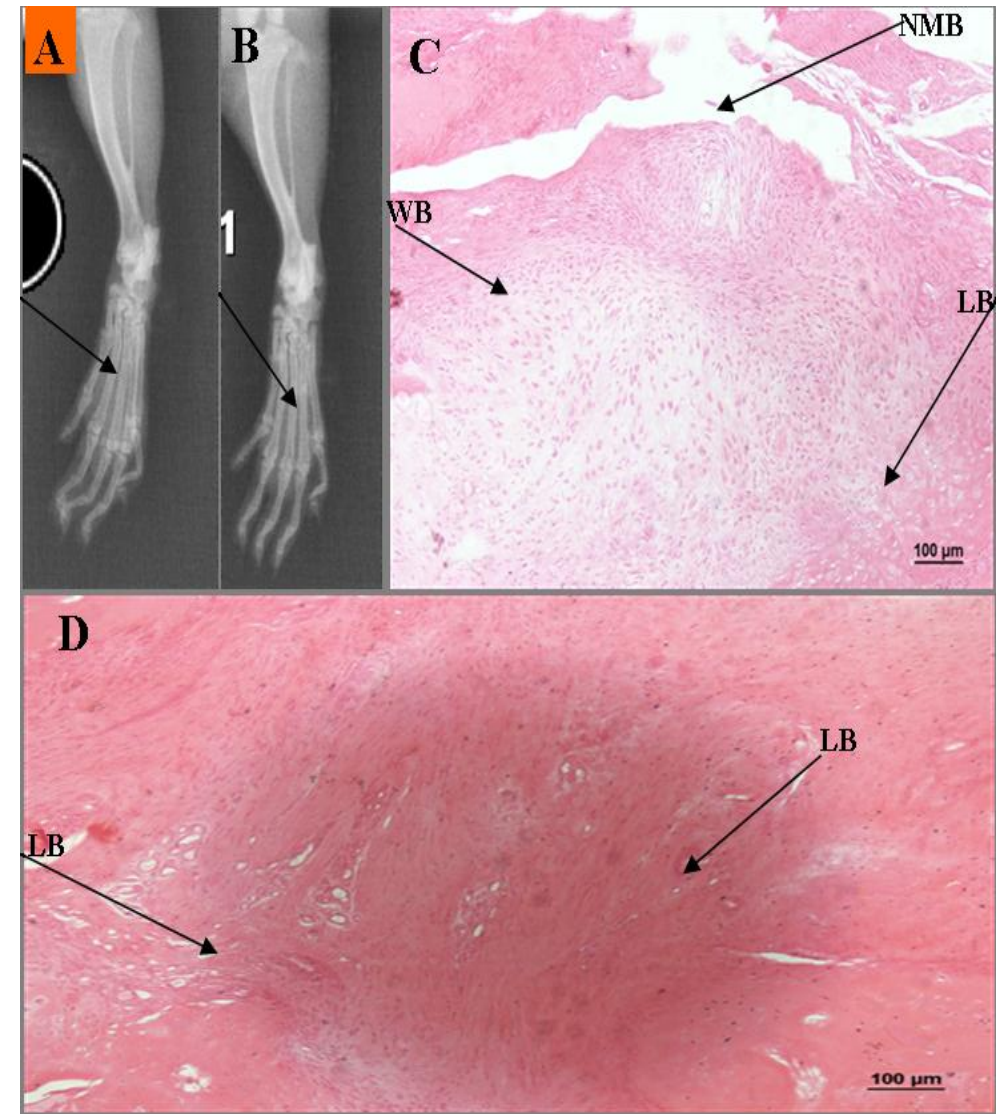

Figure 3. The representative of radiographic and light micrographic features of the fracture healing at week 5 and 7 post operation indicating non delayed union healing. (A and B) anterior posterior (AP) view of 3rd metatarsal healing at week 5 and 7 respectively, arrows indicate the fracture line bridged with mineralized bone. $(C)$, histological features at week 5 , the arrows indicates woven bone (WB), lamilar bone (LB) and non-mineralized bone (NMB). (D), histological healing at week 7, the arrows indicate predominant lamilar bone structures. Scale bar $=100 \mu \mathrm{m}$ in all panels, X10 objective. Slides were stained with $H$ and $E$

Varying degrees of weight-bearing lameness was recorded immediately after anesthetic recovery. The lameness disappeared at maximum day 6 post-operation. The maximum weight bearing score was recorded within the first 12 hrs. post-surgery. The weight bearing lameness scores subsequently declined and reaches a minimum score on day five post-surgery. By day six post-surgery, there was no weight-bearing lameness observed. There was a significant 
difference of weight-bearing scores $(\mathrm{P}<0.05$; Kruskal-Wallis one-way ANOVA) among the median scores at different healing intervals (Figure 4).

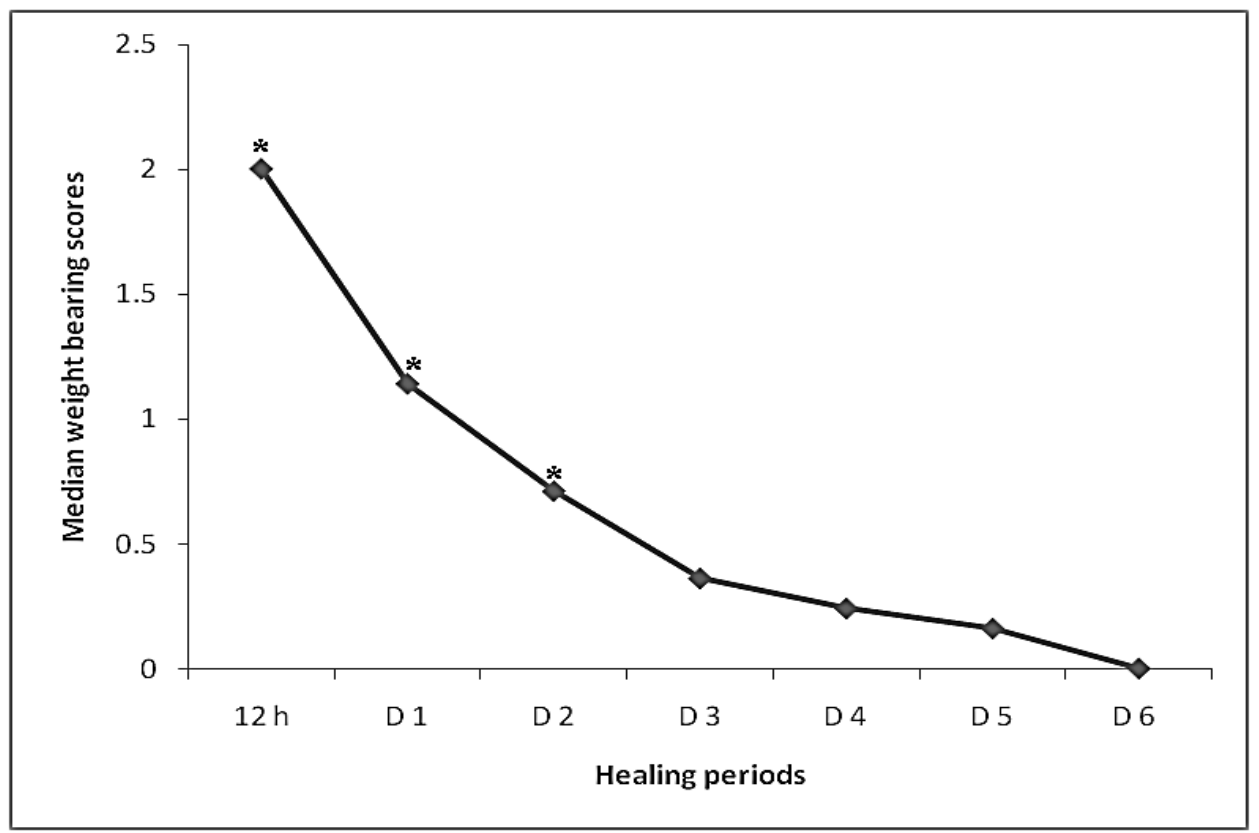

Figure 4. The line graph indicating trends of median weight bearing scores as the fracture healing progress $*$ Denotes significant differences $(P<0.05$; nonparametric Kruskal-Wallis one-way ANOVA) from D5 among median weight bearing scores at different healing intervals. $(\mathrm{n}=28)$ rats were evaluated for the weight bearing score from $12 \mathrm{hrs}$ to day 5 , whereas $(n=24)$ rats were evaluated on day 6 post surgery.

Significant variation of soft tissue damages was recorded, the maximum soft tissue damage score was observed within 24 hrs. post-surgery. The soft tissue damage score significantly declined as the healing progresses; the least score was recorded at day 10 post-surgery. On the $11^{\text {th }}$-day post-operative, there was no soft tissue damage observed (Figure 5).

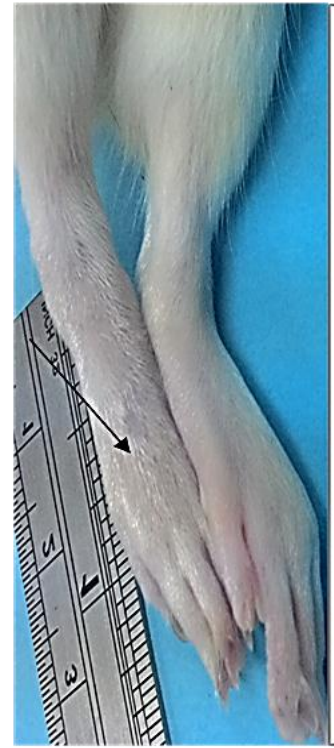

(A)

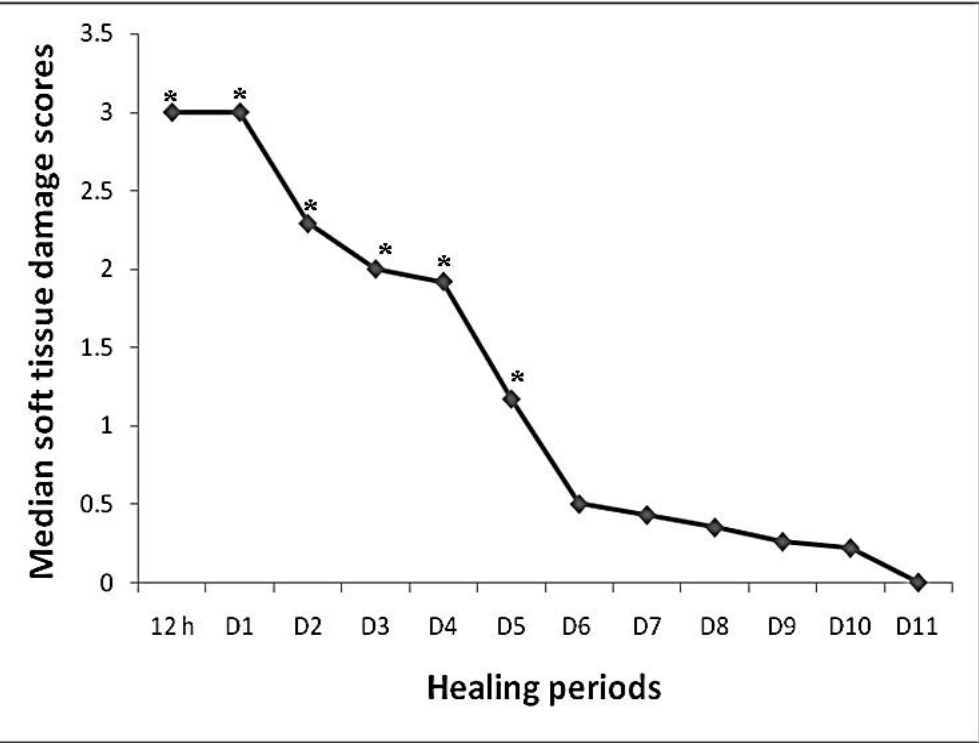

(B)

Figure 5. Photograph and graphical comparative appearance of surgical site at day 11 post surgery and graphical trend of soft tissue damage scores during healing. (A) Soft tissue injury comparison between fractured limb (indicated by arrow) and the counter lateral control limb at day eleven post operation when visible signs of soft tissue injury disappeared. (B) Trend of soft tissue damage from $12 \mathrm{hrs}$ post operation to its disappearance. *Denotes significant differences $(P<0.05$; nonparametric Kruskal-Wallis one-way ANOVA) from D10 among degree of soft tissue damage at different healing periods.

The soft tissue damage score maintains a constant peak reading between 12 to 24 hrs. postoperative. There were significant differences $(P<0.05$, non-parametric Kruskal-Wallis one-way ANOVA) in median soft tissue damage scores among the post-operative scoring intervals. 


\subsection{Pattern of Fracture Produced}

All fracture generated on the twenty-eight rats were transverse. However, $17 \%$ of the transverse fractures were anatomically located at proximal $1 / 3$ of the metatarsal shaft whereas $10 \%$ of the fractures generated were located at distal $1 / 3$ of the metatarsal shaft. Significant percentages $(73 \%)$ of the fracture produced were anatomically located at the midshaft of the metatarsal bone. There were minimal fracture angulations along the axis of the bone using anteriorposterior (AP) and lateral radiographic views taken immediately post-surgery within 0-12 hrs. after the operation. Image $\mathrm{J}$ measurement of the fracture angles revealed a greater degree of angulations in lateral view $\left(0.78 \pm 0.17^{\mathrm{O}}\right)$ compared to the anterior-posterior view $\left(0.48 \pm 0.09^{\circ}\right)$. Paired student $t$-test showed no significant difference $(\mathrm{P}>$ 0.05 ) between mean AP views angulations and L views angulations (Figure 6).

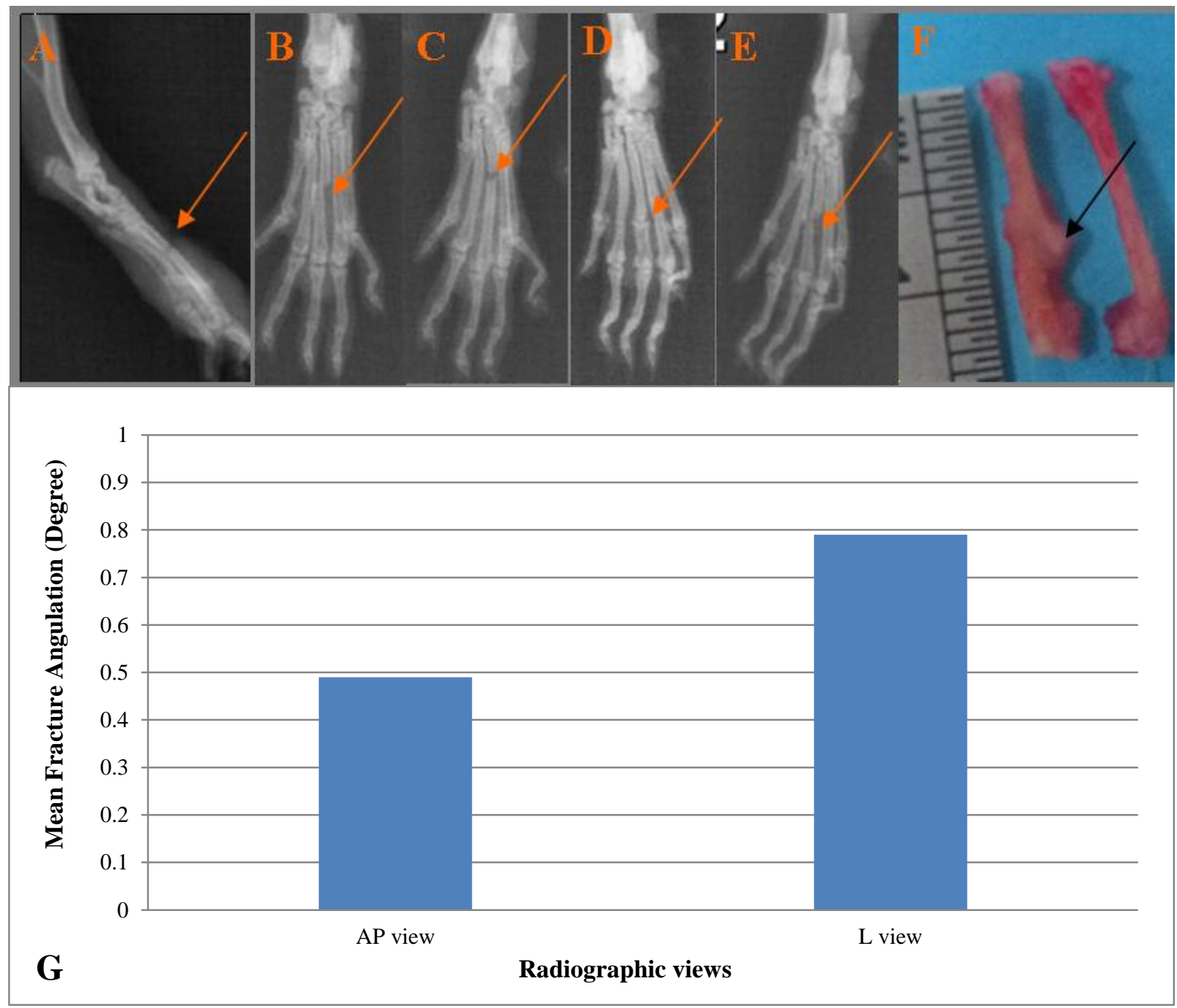

Figure 6. The radiographic, gross appearance and graphical presentation of the pattern of fracture produ ced. Lateral (L) and anteri or posterior (AP) views of representative radiographic with evidence of mid shaft fracture produced. A is a lateral view, (B, C and D) AP view radiographic with evidence of fracture angulations, (G) graphical presentation of radiographic degree of fracture angulations in lateral and anterior posterior view. There was no significant difference between two radiographic views. $A, B, C$ and $E$ radiographs were taken immediately after surgery, while $E$ was taken 1 week after surgery. $\mathrm{F}$ is a gross appearance of fractured and control bones 2 weeks after surgery showing gross angulations.

\subsection{Fracture Healing Consolidation}

The callus index (CI) was determined at 6 different points of healing intervals in order to observe the trend of fracture healing progress. It was observed that callus index was very low at day four postoperative $(1.2 \pm 0.05)$, it then significantly increased at week one $(1.97 \pm 0.09)$, and peaked at week two $(2.02 \pm 0.1)$ postoperative. The callus index then gradually starts to decline at week three, by week seven, when the experiment was terminated, it was drastically reduced to $(1.1 \pm 0.04)$. There was a significant difference $(\mathrm{P}<0.05)$ among the means callus index at weeks 1,2 and 3 when compared with initial mean index recorded at day four postoperative (Figure 7). 


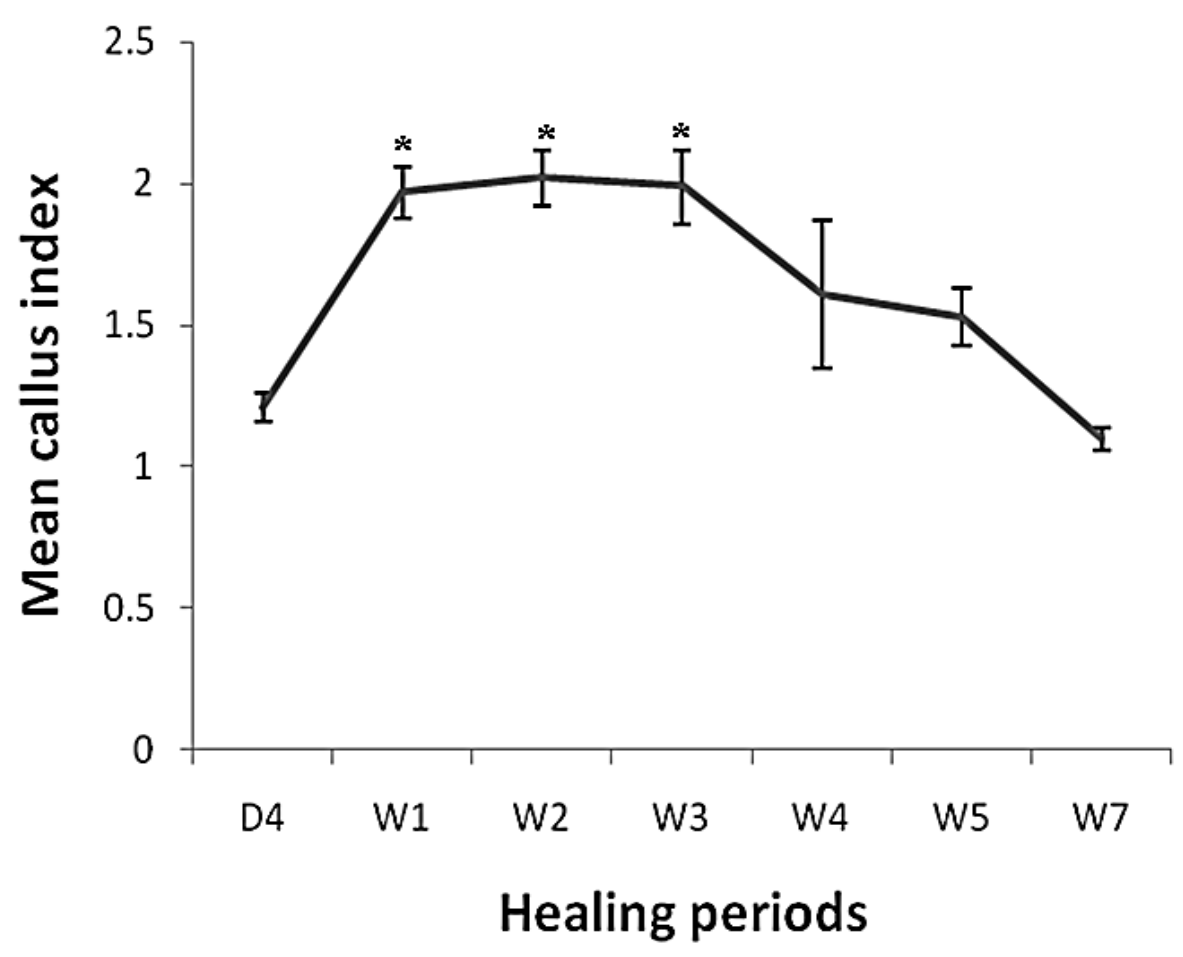

Figure 7. Graphical presentation of fracture consolidation recorded evidence by the trend of callus formation and resorption in the course of the fracture healing. *Denotes significant difference $(P<0.05$, one-way ANOVA) from D4 among the mean callus index at different healing intervals. Data were presented as Means \pm SEM.

Radiographic evidence of fracture line disappearance at different healing intervals revealed that fracture line started to disappear at week one with $10 \%$ of the radiographic fractured metatarsal has no fracture line. The percentage fracture lines disappearance progressively increased at week three, four and five post-operative. There was no fracture line observed at week seven (Table 1).

Table 1. Percentage radiographic evidence of fracture line disappearance

\begin{tabular}{cccccc}
\hline Radiograph intervals (Weeks) & W2 & W3 & W4 & W5 & W7 \\
\hline No. of rats with fracture lines appearance & 18 & 11 & 4 & 1 & 0 \\
No. of rats with fracture lines disappeared & 2 & 5 & 8 & 7 & 4 \\
$\quad$ Total no. of rats examined & 20 & 16 & 12 & 8 & 4 \\
\% of rats with fracture lines disappearance & 10 & 31.25 & 66.7 & 87.5 & 100 \\
\hline
\end{tabular}

\subsection{Radiological and Histological Healing Evaluation}

The mean healing scores based on lateral view radiograph was apparently higher than that of anterior-posterior views $3.75 \pm 0.25$ and $3.5 \pm 0.29$ out of the maximum 4 point score respectively. However, paired student $t$-test showed no significant differences between the mean scores of the two radiographic views. The mean histological healing score was $3.5 \pm 0.13$ out of the maximum 4 point score (Figure 8). 


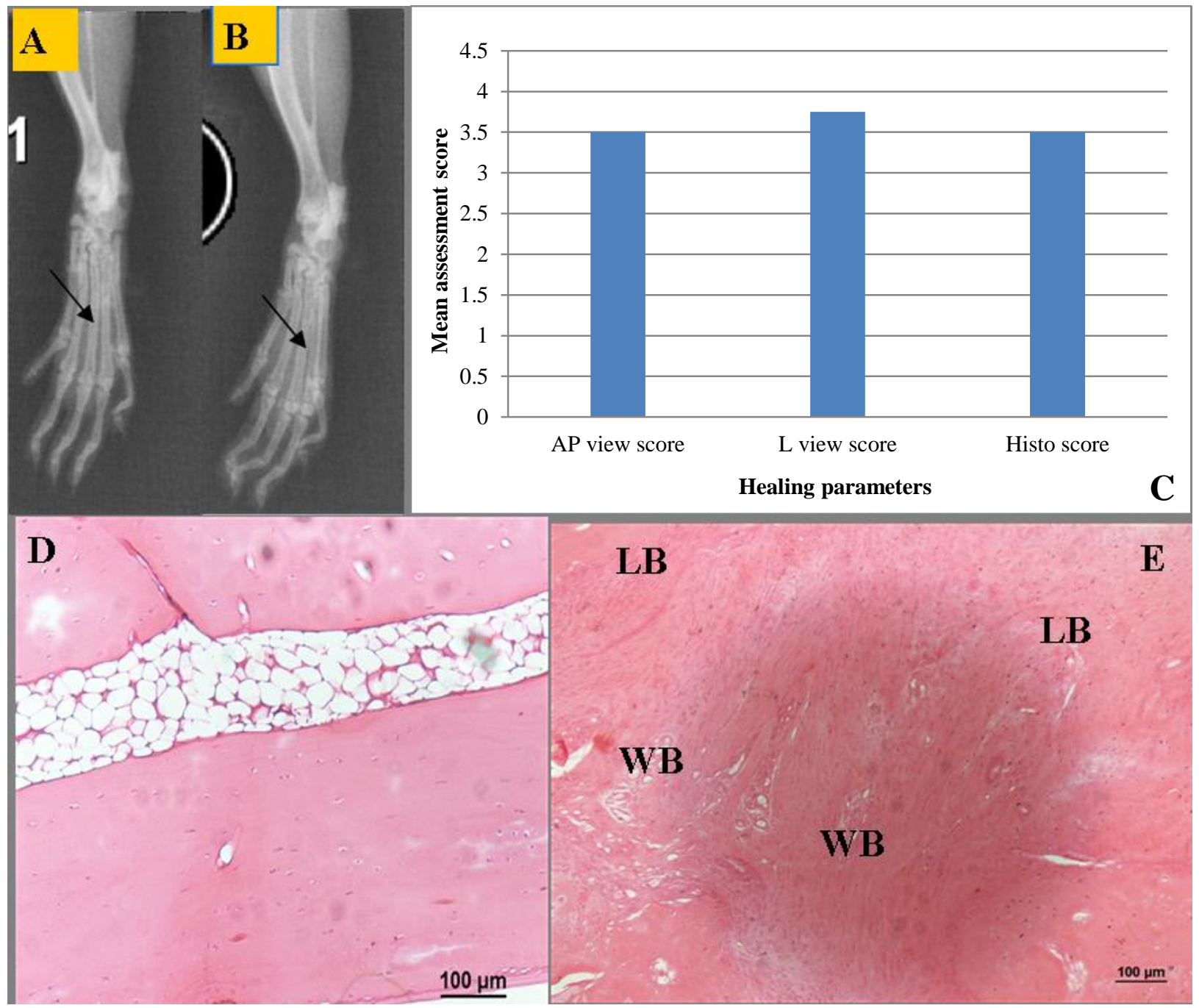

Figure 8. The radiographic, graphical and light micrographs of healing assessment at week 7 of fracture healing. (A and B) representative radiographic features of healing at week 7 , arrows on the radiographs indicate point of fractures, (C) graphical presentation of radiographic AP, $L$ views and histological healing scores showing no significant differences among the 3 healing scoring parameters. D and $E$ are histological features of controlled and fractured bones respectively at week 7 of fracture healing showing cortical bridging of the fracture line with few woven bones (WB) mixed in the predominant lamella bones (LB). Scale bar $=100 \mu \mathrm{m}$ in all panels, X10 objective. Slides were stained with $\mathrm{H}$ and E.

Kruskal-Wallis one way ANOVA shows no significant difference among the means of the anterior-posterior score, lateral views scores and the histological healing scores. The Pearson correlation coefficient between the histological and radiographic healing scores shows a positive correlation with an r-value of $(r=0.577)$.

\section{Discussions}

Most of the fracture models for biomedical investigation using rodents were performed by creating a closed fracture of either femur or tibia using various types of blunt object to generate the fracture [10, 15]. Few others were created by direct osteotomy of the femur or tibia after surgical exposure of the targeted bones [22, 37]. Creating a fracture using either blunt objects or direct osteotomy techniques in either femur or tibia regions implies traumatizing the soft tissue surrounding the bones because both regions are heavily surrounded with soft muscle tissues that play important physiological roles during movement. Therefore, it is evident that the soft tissues surrounding the fracture and the periosteum play a crucial role in the fracture healing events as reported by Claes et al. [38]; Zhang et al. [39]; li et al. [40]. Due to this reason, the use of anatomical location like metatarsal regions where there are less soft tissues mass will be preferable to avoid massive soft tissue injury that disrupt the fracture healing. Foster et al. [23] reported that apart from the disruption of fracture healing, the complications may lead to localized infection of both soft and bonny tissues around the affected area and if care is not taken it will result in septicemia.

The use of the guillotine-like device and other three-point bending apparatus to generate closed fracture models as described by numerous investigators $[10,11,12,15,19]$ have multiple recurrent cases of the animal being excluded from the studies, because the fracture produced was not at the target anatomical location proposed earlier. For 
example, $15 \%, 9 \%$ and $12 \%$ of the rats were excluded by Jackson et al. [11]; Manigrasso and O'Connor, [41]; Auregan et al. [15] respectively. In a recent study conducted by Shi et al. [20], the complications of closed tibial fracture was categorized into type I to III, with type III having the severe localized injuries ranging from muscles avulsion, vascular rupture, ischemia of distal extremities and bonny comminution. These limitations make the techniques of these popular fracture models to be a little bit difficult to be reproducible without modifications. Although the force $\left(\mathrm{Kgms}^{-2}\right)$, the mass of the object (grams) and distances (centimetre) at which the blunt object is thrown to generate the fracture at the desired anatomical location is controlled, still in few cases, the fracture is not generated at the first attempt, which makes it necessary to make several attempts in order to produce the closed fracture, this multiple attempts also affect the integrity of the soft tissues surrounding the targeted bone as reported by Auregan et al. [15].

Some of the criteria used to assess complications of fracture created from an animal model are; the inability of the animal to resume normal feed and water intake within short duration postoperative [42], degree of weight-bearing lameness and its persistence, the existence of soft tissue injury and its persistence [39]. The present study has shown that normal feed and water intake were observed to be minimally affected within the first five days postoperative, this could be associated with the stress coupled with the quantum of pain, even though analgesic was given to relieve the pain. Although, there is currently no fracture model study that quantifies the intake of feed and water as criteria for evaluating complication. This study suggested that feed and water intake could be affected within five days after generation of fracture model. This in-turn may affect the bone healing process through a decline in nutrients supply that is necessary for energy production.

Acute complete weight-bearing lameness was recorded within the first $12 \mathrm{hrs}$. and drastically reduced within 24 hrs post-operative and finally disappeared at day five postoperative. Previous literature reported acute weight-bearing lameness for up to $48 \mathrm{hrs}$. with persistence partial weight-bearing lameness for up to 2-3 weeks as normal [17]. This study revealed that partial lameness was resolved completely after day 5 post-surgery. This may suggest that our procedure has inflicted minimal complication, hence resumption of normal ambulatory at day six -post-operation. Otto et al. [17] reported persistent partial lameness until 2 weeks post-operation. The early resumption of ambulatory observed might have helped enhance the healing process of the bone. It was reported by Morshed, [43]; BighamSadegh and Oryan, [44] that, micro-motion within fracture environment due to early attempt to bear partial weight on fracture limb may bring about mechanical stimulation that can promote healing. The early weight-bearing may also prevent disuse atrophy of the fractured limb.

Moderate soft tissue injury score was recorded within the first $24 \mathrm{hrs}$. post-operation, which continues to decline progressively and completely seize at 10-day -post-operation. The continued decline of the soft tissue injury score is a strong indication that the muscle and ligaments tissues surrounding the fractured bone were not severely traumatized to the extent that the bone healing process was compromised. It was reported by Claes et al. [38] and Rotter et al. [45 that severe soft tissue injury causes disturbance of microcirculation which may interfere with inflammatory cell response by increasing permeability of the micro-vasculatures. Claes et al. [38] reported moderate soft tissue damage that persisted for more than ten-day post-operative, which brought about delayed fracture healing in their investigation. It was also reported that soft tissue trauma could delay periosteal response to healing [46, 47]. The findings of this study agreed with that of Shi et al. [20] where soft tissue injury persisted for seven days postoperative. Although the severity of the soft tissue damage they recorded was greater when compared to this study, the likely reason why they have severe soft tissue injury is that the fracture model they generated was the closed type. Therefore, compared with these existing models, our approach would have been more useful in secondary fracture healing research because we produce a model with minimal soft tissue injuries in a rat metatarsal fracture model with high reproducibility.

The anatomical location (proximal, mid-shaft or distal) of the fracture generated from the animal model and pattern of the fracture (transverse, oblique or spiral) are very important criteria to assess the quality of the fracture produced. The target of most if not all investigators, when producing a fracture model in laboratory animal is to generate transverse midshaft diaphyseal fracture of the targeted bone. Majority of the fracture generated in this study met these criteria because $100 \%$ of the fractures that we generated were transverse in the pattern. However, seventy-three per cent $(73 \%)$ of the fractures were correctly located at mid shaft. In contrast, $10 \%$ and $17 \%$ of the fractures were located more to the proximal and distal part of the metatarsal bone, respectively. The total $27 \%$ of the fracture produced out of the targeted area could be associated to the inadequate space to manipulate the $3^{\text {rd }}$ (middle metatarsal) bone because it is anatomically bounded laterally by the $2^{\text {nd }}$ and $4^{\text {th }}$ metatarsal bones, this may likely make it a bit difficult to explore the target metatarsal bone. This could be one of the limitations of this present fracture model. Li et al. [48]; Auregan et al. [15] reported that $85.8 \%$ and $88 \%$ of the femoral closed fractures produced were transverse with little deviation from the targeted mid shaft location.

Minimal fracture angulations were recorded that are more prominent on lateral views of the radiograph. This is contrary to the findings of Auregan et al. [15], where fracture angulations were prominent on the anterior, posterior 
views. However, the degree of fracture angulations we reported was lower than those recorded in a closed fracture model in which the fractured limbs were stabilized with $\mathrm{K}$-wires $[15,17]$. The high fracture angulations reported in a closed fracture model could be associated with the impact of the blunt object used for the fracture induction on the targeted bone; this is because the intramedullary pin is usually pre-inserted in place before fracture induction. The lower fracture angulations record in this study could be explained by the bilateral support of the fractured metatarsal bone provided by the $2^{\text {nd }}$ and $4^{\text {th }}$ metatarsal bone, which makes the fracture edges to be stabilized even though without an intramedullary pin or other implants support. The low degree of angulations recorded could also be associated with the rapid healing observed, along with progressive fracture consolidation recorded evidence by low callus index observed at week seventh postoperative. Many investigators are considering the high degree of fracture angulations as a deformity that may prolong the duration of fracture healing time [49]. The findings of low fracture angulations in this study corroborate with that of the study conducted by Williams et al. [19] in a mouse model where low fracture angulations were recorded in a closed femoral fracture model.

Although the aim of this study was not to keep the rats until the complete healing process has taken place, an attempt was made to assess the degree of the healing process at week seventh when animal experimentation was terminated. The histological and radiographic assessments of bone healing are among the most important qualitative and quantitative criteria used for evaluating fracture healing process as reported by Morshed, [43]; Gomez-Barrena et al. [30]; Porter et al. [32]. The radiological healing evaluation results of both lateral and anterior-posterior views revealed good, advance healing process with the majority of the radiological feature showed good bridging of the fracture gap. Similarly, quantitative histological healing scores revealed advanced healing process evidence by a small amount of cartilage within the fracture micro-environment. The high quantitative healing scores recorded both in radiological and histological assessments at week seventh signified rapid healing process, which could be associated possibly with early weight-bearing response, moderate inflammatory response linked to mild soft tissues injury recorded and possible stabilization support provided by the bilateral $2^{\text {nd }}$ and $4^{\text {th }}$ metatarsal bones. The positive degree of correlation between the histological and radiographic healing assessment is also an indication that there are agreements between the two healing assessment employed. The positive correlation found between the radiographic and histological healing assessment in this study agree with that of the study conducted by Aurégan et al.; Williams et al.; Haffner-Luntzer et al. [15, 19, 49].

\section{Conclusion}

Transverse fracture model of $3^{\text {rd }}$ metatarsal mid-shaft was successfully produced in rats. The model has minimal complications that may likely delay fracture healing or causes the death of experimental animals, hence can be reproduced. The normal feed and water intake were resumed within five days after generation of the fracture when compared with the existing models. Normal limb ambulation was also restored within five days of generation of fracture. The fracture generated has a minimal angulation that is acceptable for bone healing. The radiographic and histological healing assessments of this model indicate rapid healing devoid of any clinical complication. The major limitation of this model was the inability to produce $100 \%$ mid-shaft metatarsal fracture, because of the limited space between the $3^{\text {rd }}$ and $2^{\text {nd }}$, as well as $3^{\text {rd }}$ and $4^{\text {th }}$ metatarsal bones. Nevertheless, $73 \%$ of the open fracture generated in 28 rats that were used was targeted at the mid-shaft diaphysis of the metatarsal bone. Therefore the model could be considered to be suitable for laboratory investigation of secondary fracture healing.

\section{Funding}

This work was supported by research grant from Ministry of Higher Education Malaysia (02-01-13-1200FR).

\section{Acknowledgements}

Adamu Abdul Abubakar wants to thank Usmanu Danfodiyo Universty, Sokoto, Nigeria for granting him study leave to undertake PhD at Universiti Putra Malaysia. We would like to thank Mrs. Latifah Mohammed and Mrs. Jamilah Jahari for their invaluable help in processing of histological samples. We would like to acknowledge the assistance of technical staff of diagnostic imaging (Osman Aswari, Mohd Farid Saron and Mior Mohd Nizam) for their kind assistance in taking radiographs of the rats. We appreciate the technical assistance rendered by Drs. Lau Seng Fong and Yusuf Abba in radiographic and histological healing scoring respectively.

\section{Declaration of Competing Interest}

The authors declare that they have no known competing financial interests or personal relationships that could have appeared to influence the work reported in this paper.

\section{Ethical Approval}

The study was undertaken according to the protocol approved by the Institutional Animal Care and Used Committee of Universiti Putra Malaysia (IACUC, UPM) (Reference no. R028/2015). 


\section{References}

[1] Arvidson, K., Abdallah, B. M., Applegate, L. A., Baldini, N., Cenni, E., Gomez - Barrena, E., Granchid, D., Kassem, M., Konttinen, Y. T., Mustafa, K., Pioletti, D. P., Sillat, T. \& Finne-Wistrand, A. (2011). Bone regeneration and stem cells. Journal of cellular and molecular medicine, 15(4), 718-746. doi:10.1111/j.1582-4934.2010.01224.x.

[2] Drissi, H., \& Paglia, D. N. (2014). Surgical Procedures and Experimental Outcomes of Closed Fractures in Rodent Models. Osteoporosis and Osteoarthritis, 193-211. doi:10.1007/978-1-4939-1619-1_15.

[3] Zhu, Z.-H., Gao, Y.-S., Luo, S.-H., Zeng, B.-F., \& Zhang, C.-Q. (2011). An animal model of femoral head osteonecrosis induced by a single injection of absolute alcohol: An experimental study. Medical Science Monitor, 17(4), BR97-BR102. doi: $10.12659 / \mathrm{msm} .881708$.

[4] Mills, L. A., \& Simpson, A. H. R. W. (2012). In vivomodels of bone repair. The Journal of Bone and Joint Surgery. British Volume, 94-B(7), 865-874. doi:10.1302/0301-620x.94b7.27370.

[5] Bleedorn, J. A., Sullivan, R., Lu, Y., Nemke, B., Kalscheur, V., \& Markel, M. D. (2013). Percutaneous lovastatin accelerates bone healing but is associated with periosseous soft tissue inflammation in a canine tibial osteotomy model. Journal of Orthopaedic Research, 32(2), 210-216. doi:10.1002/jor.22502.

[6] Tunio, A., Jalila, A., Meng, G., \& Shameha, I. (2014). Experimental fracture healing with external skeletal fixation in a pigeon ulna model. Journal of Advanced Veterinary and Animal Research, 1(2), 58. doi:10.5455/javar.2014.a14.

[7] Mohamad, S., Shuid, A., Mohamed, N., Fadzilah, F., Mokhtar, S., Abdullah, S., ... Soelaiman, I. (2012). The effects of alphatocopherol supplementation on fracture healing in a postmenopausal osteoporotic rat model. Clinics, 67(9), 1077-1085. doi:10.6061/clinics/2012(09)16.

[8] Hu, J., Qu, J., Xu, D., Zhang, T., Qin, L., \& Lu, H. (2013). Combined application of low-intensity pulsed ultrasound and functional electrical stimulation accelerates bone-tendon junction healing in a rabbit model. Journal of Orthopaedic Research, 32(2), 204-209. doi:10.1002/jor.22505.

[9] Abubakar, A. A., Noordin, M. M., Azmi, T. I., Kaka, U., \& Loqman, M. Y. (2016). The use of rats and mice as animal models inex vivobone growth and development studies. Bone \& Joint Research, 5(12), 610-618. doi:10.1302/2046-3758.512.bjr-20160102.r2.

[10] Handool, K. O., Ibrahim, S. M., Kaka, U., Omar, M. A., Abu, J., Yusoff, M. S. M., \& Yusof, L. M. (2018). Optimization of a closed rat tibial fracture model. Journal of Experimental Orthopaedics, 5(1). doi:10.1186/s40634-018-0128-6.

[11] Jackson, R. W., Reed, C. A., Israel, J. A., Abou-Keer, F. K., \& Garside, H. (1970). Production of a standard experimental fracture. Canadian journal of surgery. Journal canadien de chirurgie, 13(4), 415.

[12] Bonnarens, F., \& Einhorn, T. A. (1984). Production of a standard closed fracture in laboratory animal bone. Journal of Orthopaedic Research, 2(1), 97-101. doi:10.1002/jor.1100020115.

[13] Garcia, P., Holstein, J. H., Histing, T., Burkhardt, M., Culemann, U., Pizanis, A., ... Menger, M. D. (2008). A new technique for internal fixation of femoral fractures in mice: Impact of stability on fracture healing. Journal of Biomechanics, 41(8), 1689-1696. doi:10.1016/j.jbiomech.2008.03.010.

[14] Holstein, J. H., Matthys, R., Histing, T., Becker, S. C., Fiedler, M., Garcia, P., .. Menger, M. D. (2009). Development of a Stable Closed Femoral Fracture Model in Mice. Journal of Surgical Research, 153(1), 71-75. doi:10.1016/j.jss.2008.02.042.

[15] Aurégan, J.-C., Coyle, R. M., Danoff, J. R., Burky, R. E., Akelina, Y., \& Rosenwasser, M. P. (2013). The rat model of femur fracture for bone and mineral research. Bone \& Joint Research, 2(8), 149-154. doi:10.1302/2046-3758.28.2000171.

[16] De Giacomo, A., Morgan, E. F., \& Gerstenfeld, L. C. (2014). Generation of Closed Transverse Fractures in Small Animals. Skeletal Development and Repair, 35-44. doi:10.1007/978-1-62703-989-5_3.

[17] Otto, T. E., Patka, P., \& Haarman, H. J. T. M. (1995). Closed Fracture Healing: A Rat Model. European Surgical Research, 27(4), 277-284. doi:10.1159/000129410.

[18] Schindeler, A., Mills, R. J., Bobyn, J. D., \& Little, D. G. (2017). Preclinical models for orthopedic research and bone tissue engineering. Journal of Orthopaedic Research. doi:10.1002/jor.23824.

[19] Williams, J. N., Li, Y., Valiya Kambrath, A., \& Sankar, U. (2018). The Generation of Closed Femoral Fractures in Mice: A Model to Study Bone Healing. Journal of Visualized Experiments, (138). doi:10.3791/58122.

[20] Shi, E., Chen, G., Qin, B., Yang, Y., Fang, J., Li, L., ... Gu, L. (2019). A novel rat model of tibial fracture for trauma researches: a combination of different types of fractures and soft tissue injuries. Journal of Orthopaedic Surgery and Research, 14(1). doi:10.1186/s13018-019-1386-4. 
[21] Young, K., Aquilina, A., Chesser, T. J. S., Costa, M. L., Hettiaratchy, S., Kelly, M. B., ... Woodford, M. (2019). Open tibial fractures in major trauma centres: A national prospective cohort study of current practice. Injury, 50(2), 497-502. doi:10.1016/j.injury.2018.10.028.

[22] Collier, C. D., Hausman, B. S., Zulqadar, S. H., Din, E. S., Anderson, J. M., Akkus, O., \& Greenfield, E. M. (2020). Characterization of a reproducible model of fracture healing in mice using an open femoral osteotomy. Bone Reports, 12, 100250. doi:10.1016/j.bonr.2020.100250.

[23] Foster, A. L., Moriarty, T. F., Trampuz, A., Jaiprakash, A., Burch, M. A., Crawford, R., ... Richards, R. G. (2020). Fracturerelated infection: current methods for prevention and treatment. Expert Review of Anti-Infective Therapy, 18(4), 307-321. doi:10.1080/14787210.2020.1729740.

[24] Ghiasi, M. S., Chen, J., Vaziri, A., Rodriguez, E. K., \& Nazarian, A. (2017). Bone fracture healing in mechanobiological modeling: A review of principles and methods. Bone Reports, 6, 87-100. doi:10.1016/j.bonr.2017.03.002.

[25] Bahney, C. S., Zondervan, R. L., Allison, P., Theologis, A., Ashley, J. W., Ahn, J., ... Hankenson, K. D. (2018). Cellular biology of fracture healing. Journal of Orthopaedic Research, 37(1), 35-50. doi:10.1002/jor.24170.

[26] Yakubu, A. S., Abubakar, A. A., Salihu, M. D., Jibril, A., \& Isah, I. (2010). Comparative analysis of chlorhexidine gluconate, povidone iodine and chloroxylenol as scrubbing solution. British Journal of Pharmacology and Toxicology, 1(2), 93-95.

[27] Vento, P. J, Swartz, M. E., Martin, L. B. E. \& Daniels, D. (2008). Food intake in laboratory rats provided standard and fenbendazole-supplemented diets. Journal of the American Association of the Laboratory Animal Science, 47(6), 46-50.

[28] Fitzpatrick, N., Bertran, J., \& Solano, M. A. (2014). Sliding Humeral Osteotomy: Medium-Term Objective Outcome Measures and Reduction of Complications With a Modified Technique. Veterinary Surgery, 44(2), 137-149. doi:10.1111/j.1532950x.2014.12213.x.

[29] Sylvestre, A., Wilson, J. \& Hare, J. (2002). A comparison of 2 different suture patterns for skin closure of canine ovariohysterectomy. Canadian Veterinary Journal, 43, 699-702.

[30] Gómez-Barrena, E., Rosset, P., Lozano, D., Stanovici, J., Ermthaller, C., \& Gerbhard, F. (2015). Bone fracture healing: Cell therapy in delayed unions and nonunions. Bone, 70, 93-101. doi:10.1016/j.bone.2014.07.033.

[31] Lamraski, G., Monsaert, A., De Maeseneer, M., \& Haentjens, P. (2006). Reliability and validity of plain radiographs to assess angulation of small finger metacarpal neck fractures: human cadaveric study. Journal of orthopaedic research, 24(1), 37-45. doi:10.1046/j.1365-2842.1997.00443.x.

[32] Porter, S. M., Dailey, H. L., Hollar, K. A., Klein, K., Harty, J. A., \& Lujan, T. J. (2016). Automated measurement of fracture callus in radiographs using portable software. Journal of Orthopaedic Research, 34(7), 1224-1233. doi:10.1002/jor.23146.

[33] Abubakar, A. A., Ibrahim, S. M., Ali, A. K., Handool, K. O., Khan, M. S., Noordin Mustapha, M., ... Mohamad Yusof, L. (2019). Postnatal ex vivo rat model for longitudinal bone growth investigations. Animal Models and Experimental Medicine, 2(1), 34-43. doi:10.1002/ame2.12051.

[34] Dettmeyer, R. B. (2018). Staining Techniques and Microscopy. Forensic Histopathology, 17-45. doi:10.1007/978-3-31977997-3_2.

[35] Shoji, T., Ii, M., Mifune, Y., Matsumoto, T., Kawamoto, A., Kwon, S.-M., ... Asahara, T. (2010). Local transplantation of human multipotent adipose-derived stem cells accelerates fracture healing via enhanced osteogenesis and angiogenesis. Laboratory Investigation, 90(4), 637-649. doi:10.1038/labinvest.2010.39,

[36] Estai, M. A., Soelaiman, N. I., Shuid, A. N., Das, S., Ali, A. M. \& Suhaimi, F. H. (2011). Histological changes in the fracture callus following the administration of water extract of Piper sarmentosum (Daun Kadok) in estrogen-deficient rats. Iranian Journal of Medical Science, 36(4), 281-288.

[37] Willie, B., Adkins, K., Zheng, X., Simon, U., \& Claes, L. (2009). Mechanical characterization of external fixator stiffness for a rat femoral fracture model. Journal of Orthopaedic Research, 27(5), 687-693. doi:10.1002/jor.20792.

[38] Claes, L., Maurer-Klein, N., Henke, T., Gerngross, H., Melnyk, M., \& Augat, P. (2006). Moderate soft tissue trauma delays new bone formation only in the early phase of fracture healing. Journal of Orthopaedic Research, 24(6), $1178-1185$. doi:10.1002/jor.20173.

[39] Zhang, X., Awad, H. A., O’Keefe, R. J., Guldberg, R. E., \& Schwarz, E. M. (2008). A Perspective: Engineering Periosteum for Structural Bone Graft Healing. Clinical Orthopaedics and Related Research, 466(8), 1777-1787. doi:10.1007/s11999-0080312-6.

[40] Li, H., Jiang, J., Wu, Y., \& Chen, S. (2011). Potential mechanisms of a periosteum patch as an effective and favourable approach to enhance tendon-bone healing in the human body. International Orthopaedics, 36(3), 665-669. doi:10.1007/s00264-011-1346-z. 
[41] Manigrasso, M. B., \& O’Connor, J. P. (2004). Characterization of a Closed Femur Fracture Model in Mice. Journal of Orthopaedic Trauma, 18(10), 687-695. doi:10.1097/00005131-200411000-00006.

[42] Axelrad, T. W., \& Einhorn, T. A. (2011). Use of clinical assessment tools in the evaluation of fracture healing. Injury, 42(3), 301-305. doi:10.1016/j.injury.2010.11.043.

[43] Morshed, S. (2014). Current Options for Determining Fracture Union. Advances in Medicine, 1-12. doi:10.1155/2014/708574.

[44] Bigham-Sadegh, A., \& Oryan, A. (2014). Basic concepts regarding fracture healing and the current options and future directions in managing bone fractures. International Wound Journal, 12(3), 238-247. doi:10.1111/iwj.12231.

[45] Rotter, R., Kraemer, R., Stratos, I., Vogt, P., Vollmar, B., Mittlmeier, T., \& Knobloch, K. (2012). Compartmental and muscular response to closed soft tissue injury in rats investigated by oxygen-to-see and intravital fluorescence microscopy. Journal of Trauma and Acute Care Surgery, 73(1), 73-79. doi:10.1097/ta.0b013e31824afddd.

[46] Landry, P. S., Marino, A. A., Sadasivan, K. K., \& Albright, J. A. (2000). Effect of Soft-Tissue Trauma on the Early Periosteal Response of Bone to Injury. The Journal of Trauma: Injury, Infection, and Critical Care, 48(3), 479-483. doi:10.1097/00005373-200003000-00018.

[47] Marturano, J. E., Cleveland, B. C., Byrne, M. A., O’Connell, S. L., Wixted, J. J., \& Billiar, K. L. (2008). An improved murine femur fracture device for bone healing studies. Journal of Biomechanics, 41(6), $1222-1228$. doi:10.1016/j.jbiomech.2008.01.029.

[48] Li, J., Ahmed, M., Samnegård, E., Ahmad, T., Stark, A., \& Kreicbergs, A. (2005). Spontaneous correction of angular fracture deformity in the rat. Acta Orthopaedica, 76(3), 434-441. doi:10.1080/17453670510041358.

[49] Haffner-Luntzer, M., Kovtun, A., Rapp, A. E., \& Ignatius, A. (2016). Mouse Models in Bone Fracture Healing Research. Current Molecular Biology Reports, 2(2), 101-111. doi:10.1007/s40610-016-0037-3. 